\title{
Coordination between Subway and Urban Space: A Networked Approach
}

\author{
Lei Mao ${ }^{1, *}$, Bohong Zheng ${ }^{2}$ and Cattaney James H. ${ }^{3}$ \\ ${ }^{1}$ School of Civil Engineering, Central South University, Changsha 410004, China \\ ${ }^{2}$ School of Architecture and Urban Planning, Central South University, Changsha 410004, China \\ ${ }^{3}$ Civil Engineering Research Center of NKHDL, Philadelphia, United States
}

Received 10 February 2014; Accepted 9 May 2014

\begin{abstract}
This paper selects Changsha as a case study and constructs the models of the subway network and the urban spatial network by using planning data. In the network models, the districts of Changsha are regarded as nodes and the connections between each pair of districts are regarded as edges. The method is based on quantitative analysis of the node weights and the edge weights, which are defined in the complex network theory. And the structures of subway and urban space are visualized in the form of networks. Then, through analyzing the discrepancy coefficients of the corresponding nodes and edges, the paper carries out a comparison between the two networks to evaluate the coordination. The results indicate that only $21.4 \%$ of districts and $13.2 \%$ of district connections have a rational coordination. Finally, the strategies are put forward for optimization, which suggest adjusting subway transit density, regulating land-use intensity and planning new mass transits for the uncoordinated parts.
\end{abstract}

Keywords: complex network, subway network, urban spatial network, coordination, discrepancy coefficient

\section{Introduction}

One effect of the high-speed economic growth in China is that more and more people prefer to have their own cars. But the proliferation of private cars on the road is turning cities into the nightmares of the traffic jams and the air pollution. Over the past few years, in China, hundreds of miles of subway lines have been built to address these urban issues. Even so, the new transportation is less than satisfactory. The illogicality of the subway design partly rests on the general ignorance of how the subway planning and the urban spatial planning can adapt to each other. The two plans are designed from different discipline backgrounds, which lead to the inconsistent results.

Recently, scholars in China were more concerned to the feasibility study for a subway planning. They mainly used the methods of fuzzy comprehensive evaluation method [1], grey correlation analysis method [2], data envelopment analysis [3], and entropy weight analysis method [4] for evaluation. Generally, these methods have a few defects such as the subjective results from the empirical judgment, the high-cost measure due to the lack of statistics, and the difficulty of optimizing the plans.

Many complex systems were abstracted into the complex networks such as the Internet [5], biological nets [6], food webs [7] and other areas. In the existed literatures, there are other studies that specifically have studied transportation as a complex network [8], [9], [10], including subway networks [11], [12], [13]. By analyzing the properties of a complex network, the authors explored a new approach that had investigated how to design and build more efficient and safe subway. However, these studies are

*E-mail address: feista@163.com

ISSN: 1791-2377 @ 2014 Kavala Institute of Technology. All rights reserved. generally concerned to the structure of subway networks but ignore the coordination between the subway and the urban space, which have turned the transportation into a serious urban problem.

Therefore, it is urgent to construct a more precise method of evaluating and optimizing the coordination between the subway planning and the urban spatial planning. This paper selects Changsha, the capital of Hunan province in south-central China, as the case for study. From the perspective of complex network [14], [15], the paper constructs models of the subway network and the urban spatial network. By comparing the two models, we analyze the coordination between the two plans and put forward the evaluation and the optimization of them. This is a significant study during the current large-scale construction era in China.

\section{Method descriptions}

The networks are built based on the method of graph theory. We use graph $G=(V, E)$ to express a complex network, where $V$ is a set of nodes and $E$ a set of edges. The urban districts are regarded as the nodes, as depicted by $V=\left(v_{1}, v_{2}, \cdots, v_{n}\right)$, where $n$ is the number of districts. And the connection strengths between each pair of districts are regarded as the edges, as depicted by $E=\left(e_{1}, e_{2}, \cdots, e_{m}\right)$, where $m$ is the number of edges. The connection strengths between the pairs of nodes can be fully described by a relative adjacency matrix. Therefore, the properties of network can be obtained by constructing the relative adjacency matrices and analyzing them. Finally, the 
evaluation and the optimization of the two plans are put forward by comparing the discrepancies of the properties between the subway network and the urban spatial network.

In order to apply the evaluation and the optimization in the complex network method, four steps are essential:

Step 1: divide the city into several districts, which are taken as the nodes in the subway network and the urban spatial network.

Step 2: construct the relative adjacency matrices of the two networks.

(1) Analyze the influencing factors of the nodes in the two networks.

(2) Construct the original incidence matrix by collecting the statistics of the influencing factors.

(3) Transform the original incidence matrix into the standard incidence matrix by removing the effect of dimensions on different influencing factors.

(4) Transform the incidence matrix into the absolute adjacency matrix with the cross-products method.
(5) Transform the absolute adjacency matrix into the relative matrix with the linear proportional method.

Step 3: visualize the two networks by calculating the weights of nodes and edges.

Step 4: evaluate and optimize the coordination between the two networks.

\section{Districts dividing}

According to the subway planning of Changsha, seven subway lines will be built in Changsha by 2020 , as seen in Figure 1(a). At the same time, the spatial structure of three axes and multi centres is planned for Changsha, according to the urban spatial planning, as seen in Figure 1(b). On the basis of the two plans, Changsha is divided into 14 districts-Gaoxing, Jinxia, Leifeng, Shifu, Xinshiji, Xingma, Lushan, Zhongxin, Shengfu, Guitanghe, Huangli, Konggang, Pingpu, and Muyun - which are considered as nodes in the two networks, as seen in Figure 1(c).

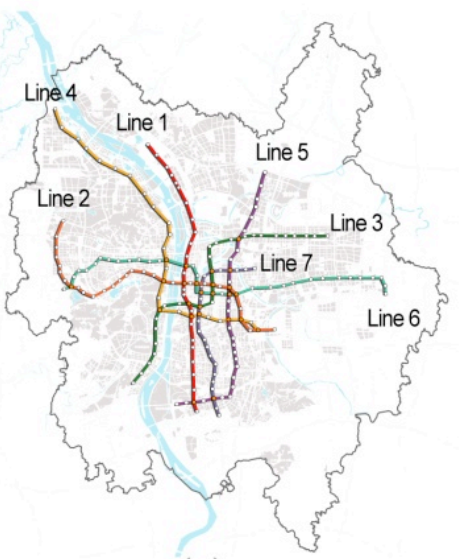

(a)

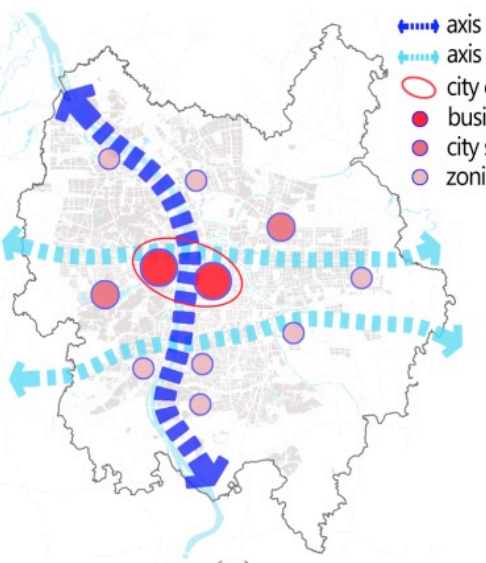

(b)

Fig.1. Districts of Changsha based on the subway planning and the urban spatial planning

\section{Matrix constructing}

Based on the related studies on the rail transportation network and the flowing space theory, the influencing factors of the subway network and the urban spatial network are put forward, as seen in Table 1.

According to Table 1, the original incidence matrix of the subway network of Changsha is constructed by collecting the planning statistics, as seen in Table 2. The matrix is defined as follows:

$$
Y_{S}=\left\{y_{i j} \mid 1 \leq i \leq n, 1 \leq j \leq f\right\}
$$

where $Y_{S}$ is the original incidence matrix of the subway network, $y_{i j}$ is the statistic of row $i$, column $j$ in matrix $Y_{S}$, the row of matrix $Y_{S}$ represents the nodes, the column of matrix $Y_{S}$ represents the influencing factors, $n$ is the number of nodes, and $f$ is the number of influencing factors of the subway network.

In order to remove the effect of dimensions on different influencing factors, the original statistical data in Table 2 is transformed into standard data (see Table 3) with the following method:

$y_{i j}{ }^{\prime}=\left\{\begin{array}{l}\frac{y_{i j}}{\sum y_{j}}, \text { if } y_{i j} \text { is a positive index } \\ \frac{y_{i j}^{-1}}{\sum y_{j}^{-1}}, \text { if } y_{i j} \text { is a negative index }\end{array}\right.$

where $y_{i j}{ }^{\prime}$ is the statistic of row $i$, column $j$ in matrix $Y_{S}{ }^{\prime}$, $y_{i j}$ is the statistic of row $i$, column $j$ in matrix $Y_{S}, \sum y_{j}$ is the sum of the statistics in column $j$ of matrix $Y_{S}$, and $\sum y_{j}^{-1}$ is the sum of the inverse of the statistics in column $j$ of matrix $Y_{S}$.

Then, we transform the standard incidence matrix into the absolute adjacency matrix (see Table 4) with the crossproducts method as follows:

$x_{i m}=\left\{\begin{array}{cl}\sum\left(y_{i j}{ }^{\prime} \cdot y_{m j}{ }^{\prime}\right), & \text { if } i \neq m \\ 0, & \text { if } i=m\end{array}\right.$ 
Lei Mao, Bohong Zheng and Cattaney James H./

Journal of Engineering Science and Technology Review 7 (2) (2014) 22 -28

Table 1. Influencing factors of the networks

\begin{tabular}{cl||cl}
\hline \multicolumn{2}{l||}{ Subway network } & Urban spatial network \\
\hline \multicolumn{1}{l||}{ Sign } & Influencing factors & Influencing factors \\
\hline$F_{S 1}$ & Number of stations on the shortcut from Line 1 & $F_{U 1}$ & Population (million people) \\
$F_{S 2}$ & Number of stations on the shortcut from Line 2 & $F_{U 2}$ & Employment opportunities (million jobs) \\
$F_{S 3}$ & Number of stations on the shortcut from Line 3 & $F_{U 3}$ & Area of construction land $\left(\mathrm{km}^{2}\right)$ \\
$F_{S 4}$ & Number of stations on the shortcut from Line 4 & $F_{U 4}$ & Number of city transportation hubs \\
$F_{S 5}$ & Number of stations on the shortcut from Line 5 & $F_{U 5}$ & Number of CBDs \\
$F_{S 6}$ & Number of stations on the shortcut from Line 6 & $F_{U 6}$ & Number of industrial parks \\
$F_{S 7}$ & Number of stations on the shortcut from Line 7 & $F_{U 7}$ & Number of main public service facilities \\
$F_{S 8}$ & Number of stations & & \\
$F_{S 9}$ & Number of transfer stations & & \\
$F_{S 10}$ & Number of subway lines & & \\
\hline
\end{tabular}

Note: $F_{S 1}-F_{S 7}$ are negative indexes, and the others are positive indexes.

Table 2. Planning statistics of the subway network of Changsha by $2020\left(Y_{S}\right)$

\begin{tabular}{|c|c|c|c|c|c|c|c|c|c|c|}
\hline & $F_{S 1}$ & $F_{S 2}$ & $F_{S 3}$ & $F_{S 4}$ & $F_{S 5}$ & $F_{S 6}$ & $F_{S 7}$ & $F_{S 8}$ & $F_{S 9}$ & $F_{S 10}$ \\
\hline$v_{1}$ & 6 & 1 & 9 & 1 & 13 & 5 & 10 & 10 & 0 & 2 \\
\hline$v_{2}$ & 1 & 8 & 11 & 7 & 13 & 6 & 10 & 7 & 0 & 1 \\
\hline$v_{3}$ & 6 & 1 & 6 & 4 & 12 & 1 & 9 & 24 & 1 & 2 \\
\hline$v_{4}$ & 2 & 1 & 4 & 1 & 9 & 1 & 6 & 9 & 1 & 3 \\
\hline$v_{5}$ & 1 & 2 & 1 & 8 & 1 & 1 & 1 & 16 & 1 & 5 \\
\hline$v_{6}$ & 10 & 3 & 1 & 5 & 4 & 1 & 6 & 11 & 0 & 2 \\
\hline$v_{7}$ & 2 & 1 & 1 & 1 & 7 & 3 & 4 & 11 & 2 & 3 \\
\hline$v_{8}$ & 1 & 1 & 1 & 1 & 2 & 1 & 1 & 35 & 11 & 6 \\
\hline$v_{9}$ & 1 & 8 & 5 & 3 & 2 & 6 & 1 & 12 & 0 & 2 \\
\hline$v_{10}$ & 4 & 1 & 2 & 1 & 1 & 1 & 1 & 21 & 6 & 5 \\
\hline$v_{11}$ & 7 & 1 & 7 & 1 & 3 & 4 & 6 & 4 & 1 & 2 \\
\hline$v_{12}$ & 15 & 8 & 11 & 10 & 9 & 1 & 11 & 7 & 0 & 1 \\
\hline$v_{13}$ & 10 & 9 & 1 & 7 & 13 & 11 & 10 & 2 & 0 & 1 \\
\hline$v_{14}$ & 1 & 12 & 9 & 7 & 1 & 13 & 1 & 11 & 2 & 3 \\
\hline
\end{tabular}

Table 3. Standard incidence matrix of the subway network of Changsha ( $Y_{S}{ }^{\prime}$

\begin{tabular}{|c|c|c|c|c|c|c|c|c|c|c|}
\hline & $F_{S 1}$ & $F_{S 2}$ & $F_{S 3}$ & $F_{S 4}$ & $F_{S 5}$ & $F_{S 6}$ & $F_{S 7}$ & $F_{S 8}$ & $F_{S 9}$ & $F_{S 10}$ \\
\hline$v_{1}$ & 0.024 & 0.119 & 0.017 & 0.134 & 0.015 & 0.024 & 0.016 & 0.056 & 0.000 & 0.053 \\
\hline$v_{2}$ & 0.143 & 0.015 & 0.014 & 0.019 & 0.015 & 0.020 & 0.016 & 0.039 & 0.000 & 0.026 \\
\hline$v_{3}$ & 0.024 & 0.119 & 0.025 & 0.034 & 0.016 & 0.121 & 0.018 & 0.133 & 0.040 & 0.053 \\
\hline$v_{4}$ & 0.072 & 0.119 & 0.038 & 0.134 & 0.021 & 0.121 & 0.027 & 0.050 & 0.040 & 0.079 \\
\hline$v_{5}$ & 0.143 & 0.060 & 0.150 & 0.017 & 0.190 & 0.121 & 0.160 & 0.089 & 0.040 & 0.132 \\
\hline$v_{6}$ & 0.014 & 0.040 & 0.150 & 0.027 & 0.048 & 0.121 & 0.027 & 0.061 & 0.000 & 0.053 \\
\hline$v_{7}$ & 0.072 & 0.119 & 0.150 & 0.134 & 0.027 & 0.040 & 0.040 & 0.061 & 0.080 & 0.079 \\
\hline$v_{8}$ & 0.143 & 0.119 & 0.150 & 0.134 & 0.095 & 0.121 & 0.160 & 0.194 & 0.440 & 0.158 \\
\hline$v_{9}$ & 0.143 & 0.015 & 0.030 & 0.045 & 0.095 & 0.020 & 0.160 & 0.067 & 0.000 & 0.053 \\
\hline$v_{10}$ & 0.036 & 0.119 & 0.075 & 0.134 & 0.190 & 0.121 & 0.160 & 0.117 & 0.240 & 0.132 \\
\hline$v_{11}$ & 0.020 & 0.119 & 0.021 & 0.134 & 0.063 & 0.030 & 0.027 & 0.022 & 0.040 & 0.053 \\
\hline$v_{12}$ & 0.010 & 0.015 & 0.014 & 0.013 & 0.021 & 0.121 & 0.015 & 0.039 & 0.000 & 0.026 \\
\hline$v_{13}$ & 0.014 & 0.013 & 0.150 & 0.019 & 0.015 & 0.011 & 0.016 & 0.011 & 0.000 & 0.026 \\
\hline$v_{14}$ & 0.143 & 0.010 & 0.017 & 0.019 & 0.190 & 0.009 & 0.160 & 0.061 & 0.080 & 0.079 \\
\hline
\end{tabular}


Lei Mao, Bohong Zheng and Cattaney James H./

Journal of Engineering Science and Technology Review 7 (2) (2014) 22 -28

Table 4. Adjacency matrix of the subway network of Changsha $\left(X_{S}\right)$

\begin{tabular}{|c|c|c|c|c|c|c|c|c|c|c|c|c|c|c|}
\hline & $v_{1}$ & $v_{2}$ & $v_{3}$ & $v_{4}$ & $v_{5}$ & $v_{6}$ & $v_{7}$ & $v_{8}$ & $v_{9}$ & $v_{10}$ & $v_{11}$ & $v_{12}$ & $v_{13}$ & $v_{14}$ \\
\hline$v_{1}$ & 0.000 & 0.012 & 0.033 & 0.045 & 0.035 & 0.021 & 0.046 & 0.064 & 0.023 & 0.056 & 0.039 & 0.011 & 0.010 & 0.021 \\
\hline$v_{2}$ & 0.012 & 0.000 & 0.016 & 0.022 & 0.038 & 0.013 & 0.023 & 0.045 & 0.030 & 0.026 & 0.012 & 0.007 & 0.006 & 0.031 \\
\hline$v_{3}$ & 0.033 & 0.016 & 0.000 & 0.049 & 0.056 & 0.036 & 0.046 & 0.097 & 0.026 & 0.074 & 0.032 & 0.025 & 0.011 & 0.028 \\
\hline$v_{4}$ & 0.045 & 0.022 & 0.049 & 0.000 & 0.064 & 0.038 & 0.062 & 0.109 & 0.035 & 0.086 & 0.047 & 0.024 & 0.016 & 0.036 \\
\hline$v_{5}$ & 0.035 & 0.038 & 0.056 & 0.064 & 0.000 & 0.068 & 0.078 & 0.166 & 0.086 & 0.139 & 0.046 & 0.032 & 0.037 & 0.106 \\
\hline$v_{6}$ & 0.021 & 0.013 & 0.036 & 0.038 & 0.068 & 0.000 & 0.047 & 0.076 & 0.026 & 0.062 & 0.023 & 0.023 & 0.028 & 0.028 \\
\hline$v_{7}$ & 0.046 & 0.023 & 0.046 & 0.062 & 0.078 & 0.047 & 0.000 & 0.138 & 0.041 & 0.099 & 0.050 & 0.017 & 0.032 & 0.045 \\
\hline$v_{8}$ & 0.064 & 0.045 & 0.097 & 0.109 & 0.166 & 0.076 & 0.138 & 0.000 & 0.091 & 0.256 & 0.083 & 0.038 & 0.040 & 0.131 \\
\hline$v_{9}$ & 0.023 & 0.030 & 0.026 & 0.035 & 0.086 & 0.026 & 0.041 & 0.091 & 0.000 & 0.076 & 0.027 & 0.013 & 0.014 & 0.074 \\
\hline$v_{10}$ & 0.056 & 0.026 & 0.074 & 0.086 & 0.139 & 0.062 & 0.099 & 0.256 & 0.076 & 0.000 & 0.074 & 0.034 & 0.027 & 0.110 \\
\hline$v_{11}$ & 0.039 & 0.012 & 0.032 & 0.047 & 0.046 & 0.023 & 0.050 & 0.083 & 0.027 & 0.074 & 0.000 & 0.012 & 0.011 & 0.032 \\
\hline$v_{12}$ & 0.011 & 0.007 & 0.025 & 0.024 & 0.032 & 0.023 & 0.017 & 0.038 & 0.013 & 0.034 & 0.012 & 0.000 & 0.006 & 0.014 \\
\hline$v_{13}$ & 0.010 & 0.006 & 0.011 & 0.016 & 0.037 & 0.028 & 0.032 & 0.040 & 0.014 & 0.027 & 0.011 & 0.006 & 0.000 & 0.013 \\
\hline$v_{14}$ & 0.021 & 0.031 & 0.028 & 0.036 & 0.106 & 0.028 & 0.045 & 0.131 & 0.074 & 0.110 & 0.032 & 0.014 & 0.013 & 0.000 \\
\hline
\end{tabular}

Table 5. Relative adjacency matrix of the subway network of Changsha $\left(X_{S}{ }^{\prime}\right)$

\begin{tabular}{|c|c|c|c|c|c|c|c|c|c|c|c|c|c|c|}
\hline & $v_{1}$ & $v_{2}$ & $v_{3}$ & $v_{4}$ & $v_{5}$ & $v_{6}$ & $v_{7}$ & $v_{8}$ & $v_{9}$ & $v_{10}$ & $v_{11}$ & $v_{12}$ & $v_{13}$ & $v_{14}$ \\
\hline$v_{1}$ & 0.000 & 0.261 & 0.696 & 0.944 & 0.740 & 0.447 & 0.962 & 1.341 & 0.473 & 1.171 & 0.819 & 0.231 & 0.204 & 0.430 \\
\hline$v_{2}$ & 0.261 & 0.000 & 0.328 & 0.466 & 0.803 & 0.262 & 0.480 & 0.940 & 0.633 & 0.549 & 0.246 & 0.151 & 0.135 & 0.652 \\
\hline$v_{3}$ & 0.696 & 0.328 & 0.000 & 1.027 & 1.163 & 0.762 & 0.955 & 2.020 & 0.541 & 1.546 & 0.673 & 0.513 & 0.231 & 0.588 \\
\hline$v_{4}$ & 0.944 & 0.466 & 1.027 & 0.000 & 1.349 & 0.805 & 1.296 & 2.274 & 0.739 & 1.805 & 0.984 & 0.506 & 0.324 & 0.763 \\
\hline$v_{5}$ & 0.740 & 0.803 & 1.163 & 1.349 & 0.000 & 1.414 & 1.621 & 3.476 & 1.788 & 2.913 & 0.961 & 0.677 & 0.769 & 2.211 \\
\hline$v_{6}$ & 0.447 & 0.262 & 0.762 & 0.805 & 1.414 & 0.000 & 0.983 & 1.599 & 0.552 & 1.297 & 0.488 & 0.478 & 0.591 & 0.581 \\
\hline$v_{7}$ & 0.962 & 0.480 & 0.955 & 1.296 & 1.621 & 0.983 & 0.000 & 2.894 & 0.848 & 2.075 & 1.038 & 0.351 & 0.668 & 0.937 \\
\hline$v_{8}$ & 1.341 & 0.940 & 2.020 & 2.274 & 3.476 & 1.599 & 2.894 & 0.000 & 1.905 & 5.352 & 1.726 & 0.787 & 0.843 & 2.740 \\
\hline$v_{9}$ & 0.473 & 0.633 & 0.541 & 0.739 & 1.788 & 0.552 & 0.848 & 1.905 & 0.000 & 1.588 & 0.554 & 0.279 & 0.291 & 1.548 \\
\hline$v_{10}$ & 1.171 & 0.549 & 1.546 & 1.805 & 2.913 & 1.297 & 2.075 & 5.352 & 1.588 & 0.000 & 1.540 & 0.708 & 0.572 & 2.294 \\
\hline$v_{11}$ & 0.819 & 0.246 & 0.673 & 0.984 & 0.961 & 0.488 & 1.038 & 1.726 & 0.554 & 1.540 & 0.000 & 0.244 & 0.230 & 0.676 \\
\hline$v_{12}$ & 0.231 & 0.151 & 0.513 & 0.506 & 0.677 & 0.478 & 0.351 & 0.787 & 0.279 & 0.708 & 0.244 & 0.000 & 0.118 & 0.291 \\
\hline$v_{13}$ & 0.204 & 0.135 & 0.231 & 0.324 & 0.769 & 0.591 & 0.668 & 0.843 & 0.291 & 0.572 & 0.230 & 0.118 & 0.000 & 0.277 \\
\hline$v_{14}$ & 0.430 & 0.652 & 0.588 & 0.763 & 2.211 & 0.581 & 0.937 & 2.740 & 1.548 & 2.294 & 0.676 & 0.291 & 0.277 & 0.000 \\
\hline
\end{tabular}

Table 6. Planning statistics of the urban space of Changsha by $2020\left(Y_{U}\right)$

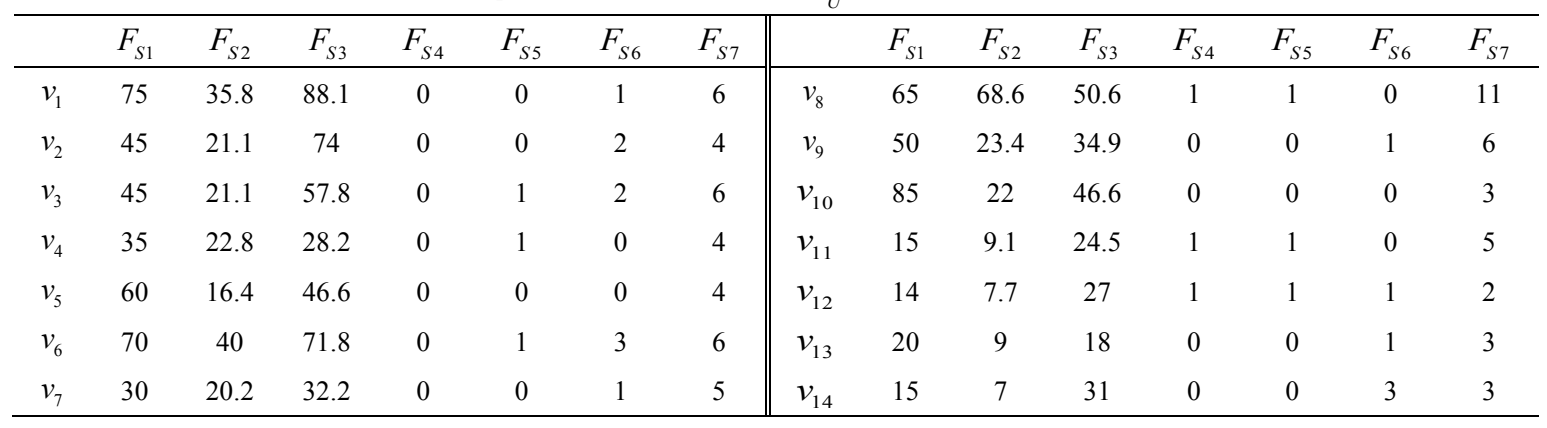

where $x_{i m}$ is the statistic of row $i$, column $m$ in matrix $X_{S}$, and $y_{i j}{ }^{\prime}, y_{m j}{ }^{\prime} \in\left\{Y_{S}{ }^{\prime}\right\}$.

To facilitate the comparison, the absolute adjacency matrix is transformed into the relative matrix (see Table 5) with the linear proportional method:

$$
x_{i j}{ }^{\prime}=\frac{n(n-1) x_{i j}}{\sum x_{i j}}
$$

where $x_{i j}{ }^{\prime}$ is the statistic of row $i$, column $j$ in matrix $X_{S}{ }^{\prime}, x_{i j} \in\left\{X_{S}\right\}, n$ is the number of nodes in the network, and $n=14$. 
Lei Mao, Bohong Zheng and Cattaney James H./

Journal of Engineering Science and Technology Review 7 (2) (2014) 22 -28

With the same steps as the matrix constructing of the subway network, we collect the planning statistics of the urban space of Changsha, as seen in Table 6. And the statistics are transformed into the relative adjacency matrix, as seen in Table 7.

Table 7. Relative adjacency matrix of the urban space of Changsha $\left(X_{U}{ }^{\prime}\right)$

\begin{tabular}{ccccccccccccccc}
\hline & $v_{1}$ & $v_{2}$ & $v_{3}$ & $v_{4}$ & $v_{5}$ & $v_{6}$ & $v_{7}$ & $v_{8}$ & $v_{9}$ & $v_{10}$ & $v_{11}$ & $v_{12}$ & $v_{13}$ & $v_{14}$ \\
\hline$v_{1}$ & 0.000 & 1.405 & 1.375 & 0.787 & 0.991 & 1.946 & 0.932 & 1.862 & 1.140 & 1.155 & 0.543 & 0.556 & 0.584 & 0.891 \\
$v_{2}$ & 1.405 & 0.000 & 1.309 & 0.526 & 0.678 & 1.861 & 0.811 & 1.220 & 0.942 & 0.774 & 0.377 & 0.571 & 0.575 & 1.158 \\
$v_{3}$ & 1.375 & 1.309 & 0.000 & 1.387 & 0.673 & 2.694 & 0.837 & 2.145 & 0.978 & 0.756 & 1.256 & 1.407 & 0.592 & 1.159 \\
$v_{4}$ & 0.787 & 0.526 & 1.387 & 0.000 & 0.477 & 1.609 & 0.415 & 1.870 & 0.523 & 0.556 & 1.128 & 1.043 & 0.231 & 0.232 \\
$v_{5}$ & 0.991 & 0.678 & 0.673 & 0.477 & 0.000 & 0.929 & 0.482 & 1.097 & 0.626 & 0.746 & 0.332 & 0.250 & 0.279 & 0.292 \\
$v_{6}$ & 1.946 & 1.861 & 2.694 & 1.609 & 0.929 & 0.000 & 1.175 & 2.700 & 1.375 & 1.091 & 1.361 & 1.640 & 0.834 & 1.665 \\
$v_{7}$ & 0.932 & 0.811 & 0.837 & 0.415 & 0.482 & 1.175 & 0.000 & 1.037 & 0.671 & 0.540 & 0.312 & 0.344 & 0.377 & 0.655 \\
$v_{8}$ & 1.862 & 1.220 & 2.145 & 1.870 & 1.097 & 2.700 & 1.037 & 0.000 & 1.285 & 1.263 & 4.928 & 4.688 & 0.566 & 0.551 \\
$v_{9}$ & 1.140 & 0.942 & 0.978 & 0.523 & 0.626 & 1.375 & 0.671 & 1.285 & 0.000 & 0.722 & 0.382 & 0.392 & 0.440 & 0.711 \\
$v_{10}$ & 1.155 & 0.774 & 0.756 & 0.556 & 0.746 & 1.091 & 0.540 & 1.263 & 0.722 & 0.000 & 0.343 & 0.277 & 0.313 & 0.313 \\
$v_{11}$ & 0.543 & 0.377 & 1.256 & 1.128 & 0.332 & 1.361 & 0.312 & 4.928 & 0.382 & 0.343 & 0.000 & 4.369 & 0.179 & 0.192 \\
$v_{12}$ & 0.556 & 0.571 & 1.407 & 1.043 & 0.250 & 1.640 & 0.344 & 4.688 & 0.392 & 0.277 & 4.369 & 0.000 & 0.253 & 0.540 \\
$v_{13}$ & 0.584 & 0.575 & 0.592 & 0.231 & 0.279 & 0.834 & 0.377 & 0.566 & 0.440 & 0.313 & 0.179 & 0.253 & 0.000 & 0.548 \\
$v_{14}$ & 0.891 & 1.158 & 1.159 & 0.232 & 0.292 & 1.665 & 0.655 & 0.551 & 0.711 & 0.313 & 0.192 & 0.540 & 0.548 & 0.000 \\
\hline
\end{tabular}

Table 8. Node weights of the two models

\begin{tabular}{|c|c|c|c|c|c|c|c|c|c|c|c|c|c|c|}
\hline & $v_{1}$ & $v_{2}$ & $v_{3}$ & $v_{4}$ & $v_{5}$ & $v_{6}$ & $v_{7}$ & $v_{8}$ & $v_{9}$ & $v_{10}$ & $v_{11}$ & $v_{12}$ & $v_{13}$ & $v_{14}$ \\
\hline$S_{s i}$ & 8.72 & 5.91 & 11.04 & 13.28 & 19.88 & 10.26 & 15.11 & 27.90 & 11.74 & 23.41 & 10.18 & 5.33 & 5.25 & 13.99 \\
\hline$S_{u i}$ & 14.17 & 12.21 & 16.57 & 10.78 & 7.85 & 20.88 & 8.59 & 25.21 & 10.19 & 8.85 & 15.70 & 16.33 & 5.77 & 8.91 \\
\hline
\end{tabular}

Note: $S_{s i}$ and $S_{u i}$ indicate the node weight of $v_{i}$ in the two models respectively.

\section{Models visualizing}

The connection strength between node $v_{i}$ and node $v_{j}$ is defined as edge weight, as formulated by $w_{i j}$. From the view of the complex network theory, the connection strengths between the pairs of nodes can be fully described by a relative adjacency matrix, as depicted by $X^{\prime}=\left\{x_{i j}{ }^{\prime} \mid 1 \leq i, j \leq n\right\}$, where $n$ is the number of nodes. According to the matrix constructing, the formula of $w_{i j}$ is defined as follows:

$$
w_{i j}=x_{i j}^{\prime}
$$

where $x_{i j}{ }^{\prime}$ is the statistic of row $i$, column $j$ in the relative adjacency matrix (see Table 5 and Table 7).

Corresponding to edge weight, the strength of node $v_{i}$ is defined as node weight, as formulated by $S_{i}$. The formula of $S_{i}$ is defined as follows:

$$
S_{i}=\sum_{j \in N_{i}} w_{i j}
$$

where $N_{i}$ is the set of nodes adjacent to node $v_{i}$.

The calculations of node weights in the two models are shown in Table 8.

With the radius represented by node weight and line width represented by edge weight, the models can be visualized in the form of networks, as seen in Figure 2 and Figure 3.

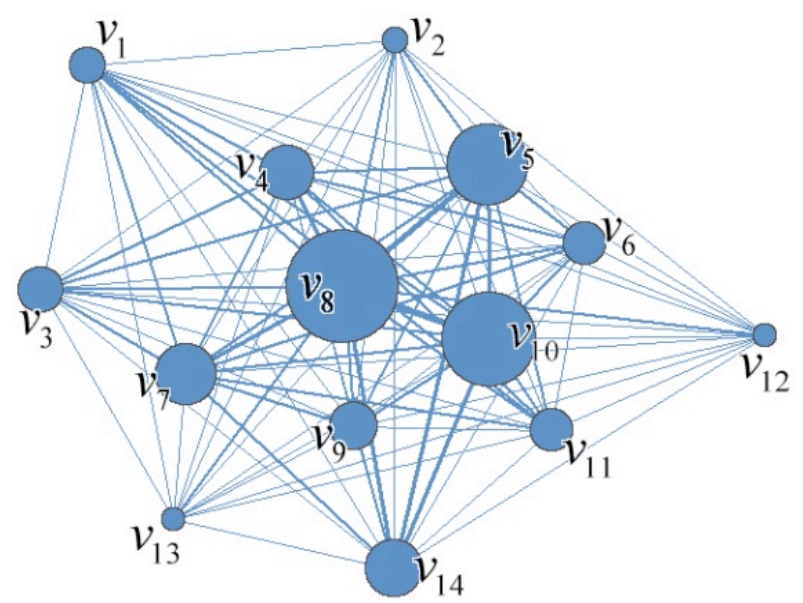

Fig. 2. Subway network of Changsha 


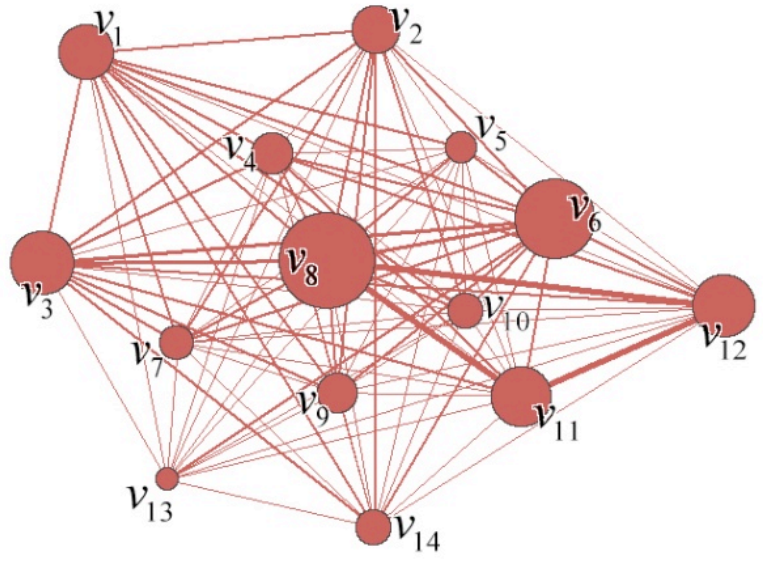

Fig. 3. Urban spatial network of Changsha

\section{Evaluation and optimization}

The discrepancies between the two graphs are obvious, which are caused by the improper coordination between the subway planning and the urban spatial planning. The quantitative evaluation of the coordination can be carried out by analyzing the discrepancy coefficients of the corresponding nodes and edges in the two networks. The formulae of the discrepancy coefficients are defined as follows:

$$
D_{i}=\left|\frac{S_{s i}-S_{u i}}{S_{s i}+S_{u i}}\right|
$$

where $D_{i}$ is the node discrepancy coefficient of node $v_{i}$, $S_{s i}$ is the node weight of node $v_{i}$ in the subway network, and $S_{u i}$ is the node weight of node $v_{i}$ in the urban spatial network (see Table 8).

$$
D_{i j}=\left|\frac{w_{s i j}-w_{u i j}}{w_{s i j}+w_{u i j}}\right|
$$

where $D_{i j}$ is the edge discrepancy coefficient of the edge between node $v_{i}$ and node $v_{j}, w_{s i j}$ is the edge weight between node $v_{i}$ and node $v_{j}$ in the subway network (see Table 5), and $w_{u i j}$ is the edge weight between node $v_{i}$ and node $v_{j}$ in the urban spatial network (see Table 7).

The index ranges from 0 to 1 , and a lower coefficient indicates that the coordination is more suitable between the two networks. The evaluations of coordination by analyzing the discrepancy coefficients are shown in Figure 4 and Figure 5.

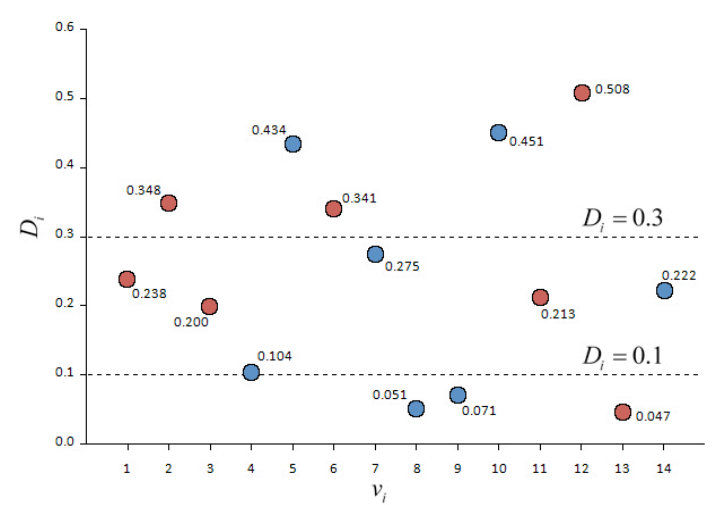

Fig. 4. Node evaluation according to discrepancy coefficients

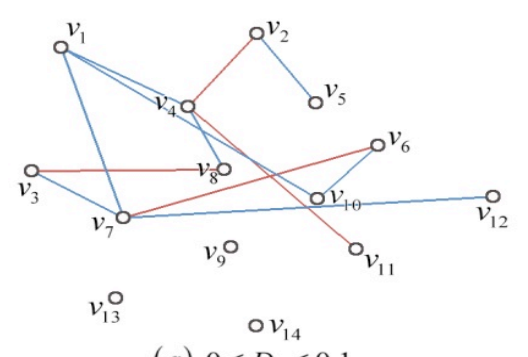

(a) $0 \leq D_{i j} \leq 0.1$

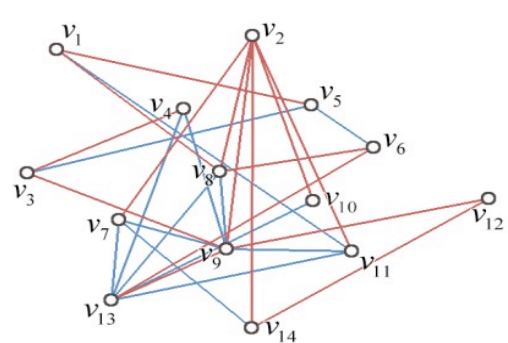

(b) $0.1<D_{i j} \leq 0.3$

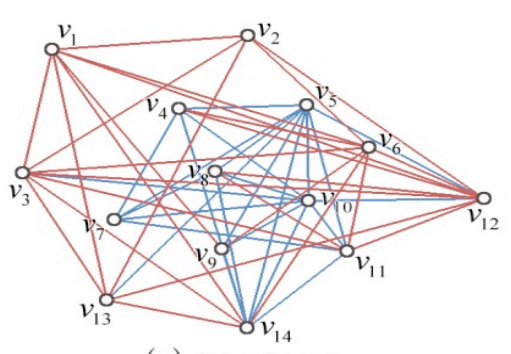

(c) $0.3<D_{i j} \leq 1$

Fig. 5. Edge evaluation according to discrepancy coefficients

As seen in the two figures, nodes and edges with higher weights in the subway network $\left(S_{s i}>S_{u i}\right.$ or $\left.w_{s i j}>w_{u i j}\right)$ appear in blue, and nodes and edges with higher weights in the urban spatial network $\left(S_{s i}<S_{u i}\right.$ or $\left.w_{s i j}<w_{u i j}\right)$ appear in red. The evaluations of coordination are divided into three: suitable coordination $\left(0 \leq D_{i}, D_{i j} \leq 0.1\right)$, medium coordination $\left(0.1<D_{i}, D_{i j} \leq 0.3\right)$ and improper coordination $\left(0.3<D_{i}, D_{i j} \leq 1\right)$.

As seen in Figure 4, the nodes of $v_{8}, v_{9}$ and $v_{13}$ are in the part of suitable coordination, whose $D_{i}$ ranges from 0 to 0.1 . The nodes of $v_{1}, v_{3}, v_{4}, v_{7}, v_{11}$ and $v_{14}$ are medium, whose $D_{i}$ ranges from 0.1 to 0.3 . And the nodes of $v_{2}, v_{5}, v_{6}, v_{10}$ and $v_{12}$ are improper, whose $D_{i}$ ranges from 0.3 to 1. As seen in Figure 5(a), the edges between the pairs of $v_{1}-v_{4}, v_{1}-v_{7}, v_{1}-v_{10}, v_{2}-v_{4}, v_{2}-v_{5}, v_{3}-v_{7}$, $v_{3}-v_{8}, v_{4}-v_{8}, v_{4}-v_{11}, v_{6}-v_{7}, v_{6}-v_{10}$ and $v_{7}-v_{12}$ are in the part of suitable coordination, whose $D_{i j}$ ranges from 0 to 0.1. As seen in Figure 5(b), 28 edges are medium, whose $D_{i j}$ ranges from 0.1 to 0.3 . And 51 edges are improper, 
whose $D_{i j}$ ranges from 0.3 to 1 , as seen in Figure 5(c). That is to say, only $21.4 \%$ of districts and $13.2 \%$ of connection strengths have a rational coordination, and $35.7 \%$ of districts and $56.1 \%$ of connection strengths are uncoordinated, from the evaluation of the two graphs.

Through evaluating the coordination between the subway and urban space of Changsha, the optimization strategies are put forward in following aspects:

(1) The districts of Xinshiji, Lushan and Guitanghe, where $S_{s i}>S_{u i}$, have obviously higher connection strengths with most other districts in the subway network. Accordingly, it is necessary to increase land-use intensity appropriately in the districts above.

(2) Slightly decrease the density of subway transit in Muyun, which is in the periphery of south Changsha and whose node weight in the subway network is slightly higher than in the urban spatial network.

(3) The districts of Jinxia, Xingma and Konggang, where $S_{s i}<S_{u i}$, have obviously higher connection strengths with most other districts in the urban spatial network. Accordingly, it is necessary to increase the density of subway transit appropriately in the three districts.

(4) Slightly decrease land-use intensity in Gaoxing and Leifeng, which are in the periphery of north-west Changsha and whose node weights in the subway network are slightly lower than in the urban spatial network.

(5) The edge weights of Jinxia-Xingma, ZhongxinHuangli, Huangli-Konggang, Gaoxing-Jinxia, GaoxingXingma and Jinxia-Pingpu in the subway network are obviously lower than in the urban spatial network. Accordingly, it is necessary to plan new mass transits, such as bus rapid transits or bus-only lanes between these pairs of districts.

\section{Conclusions}

The coordination between subway and urban space is crucial for effective transportation, dynamic economy and rational land-use in cities. However, the subway planning and urban spatial planning are designed from different discipline backgrounds, which leads to the generally uncoordinated results. In this context, it is significative to construct a method to link the two plans and put forward the evaluation and the optimization of the coordination between them.

From the perspective of a complex network, this study constructs the models of the subway network and the urban spatial network of Changsha. We divide Changsha into 14 districts, which are considered as nodes in the two networks. And the connections among the districts are regarded as edges. By analyzing the node weights and edge weights of the networks, the data visualization of the two models is realized. To study the coordination between the subway planning and the urban spatial planning, the discrepancy coefficients of the corresponding nodes and node connections between the models are figured out. Finally, the evaluation and the optimization of the two plans are put forward, according to the results of discrepancy coefficients.

The evaluation results indicate that most districts and district connections are uncoordinated between the subway and the urban space in Changsha. Furthermore, the optimization strategies of planning adjustment are proposed, which enables the subway and the urban space to adapt to each other.

This method avoids the shortcomings of strong subjectivity, high-cost evaluation, and elusive optimization in previous related researches. However, this paper ignores the effects of subway management on the subway planning and the spatial planning, which can be improved in future study to achieve a more precise and holistic method for evaluation and optimization.

\section{Acknowledgment}

This work was supported by Graduate Innovative Research Project of Hunan Province No. CX2011B100.

\section{References}

1. Junfang, L., Xiaoping, W., "Synthetic evaluation for urban rail transit line network planning scheme based on AHP-Fuzzy method", Journal of Wuhan University of Technology, 31 (2), 2007, pp. 205208. (in Chinese)

2. Yuling, Y., Ling, J., "Study on synthetic evaluation of inter-city rail transit planning", Journal of the China Railway Society, 27 (5), 2005, pp. 24-30. (in Chinese)

3. Yuzhao, Z., Yusong, Y., Nan, J., Hongwei, Z., "Research on the comprehensive evaluation of urban rail transit network plan with DEA method", Journal of Railway Engineering Society, 8 (8), 2010, pp. 96-99. (in Chinese)

4. Jing, L., Hao, M., Ling, W., "Comprehensive evaluation research of city rail transit network planning based on the model of matter element and entropy weight", Journal of engineering management, 27 (1), 2013, pp. 19-23. (in Chinese)

5. Barabasi, A. L., Albert, R., Jeong, H., "Mean-field theory for scalefree random networks", Physica A, 272 (1-2), 1999, pp. 173 - 187.

6. Dorogovtsev, S.N., Mendes, J.F.F., Samukhin, A.N., "Principles of statistical mechanics of uncorrelated random networks", Nuclear Physics B,666 (3), 2003, pp. 396-416.

7. Montoya, J.M., Solé, R.V., "Small World Patterns in Food Webs", Journal of Theoretical Biology, 214(3), 2002, pp. 405-412.
8. Zadeh, A.S.M., Rajabi, M.A., "Analyzing the effect of the street network configuration on the efficiency of an urban transportation system", Cities, 31, 2013, pp. 285-297.

9. Meisel, F., Kirschstein, T., Bierwirth, C., "Integrated production and intermodal transportation planning in large scale productiondistribution-networks", Transportation Research Part E: Logistics and Transportation Review, 60, 2013, pp. 62-78.

10. Wei, P., Chen, L., Sun, D., "Algebraic connectivity maximization of an air transportation network: The flight routes' addition/deletion problem", Transportation Research Part E: Logistics and Transportation Review, 61, 2014, pp. 13-27.

11. Angeloudis, P., Fisk, D., "Large subway systems as complex networks", Physica A, 367, 2006, pp. 553-558.

12. Barberillo, J., Saldana, J., "Navigation in large subway networks: An informational approach", Physica A, 390 (2), 2011, pp. 374386.

13. Jianhua, Z., Xiaoming, X., Liu, H., Shuliang, W., Qi, F., "Networked analysis of the Shanghai subway network, in China", Physica A, 390 (23-24), 2011, pp. 4562-4570.

14. Callaway, D.S., Newman, M.E.J, Strogatz, S.H., Watts, D.J., "Network robustness and fragility: Percolation on random graphs", Physical Review Letters, 85 (25), 2000, pp. 5468-5471.

15. Albert, R., Barabasi, A.L., "Statistical mechanics of complex networks", Reviews of Modern Physics, 74 (1), 2002, pp. 47-97. 